\title{
Occurrence of concurrent infections with multiple serotypes of dengue viruses during 2013 to 2015 in northern Kerala, India
}

\author{
Manchala Nageswar Reddy ${ }^{1}$, Ranjeet Dungdung ${ }^{1}$, Lathika Valliyott $^{1}$ ， Rajendra Pilankatta ${ }^{\text {Corresp. }}{ }^{2}$ \\ 1 Department of Biochemistry and Molecular Biology, Central University of Kerala, kasargod, kerala, India \\ 2 Department of Biochemistry and Molecular Biology, Central University of Kerala, kasargod, kerala, India \\ Corresponding Author: Rajendra Pilankatta \\ Email address: praj74@cukerala.ac.in
}

Background: Dengue is a global human public health threat, causing severe morbidity and mortality. The occurrence of sequential infection by more than one serotype of dengue virus (DENV) is a major contributing factor for the induction of Dengue hemorrhagic fever [DHF] and Dengue shock syndrome [DSS], two major medical conditions caused by DENV infection. However, there is no specific drug or vaccine available against dengue infection. There are reports indicating the increased incidence of concurrent infection of dengue in several tropical and subtropical regions. Recently, increasing number of DHF and DSS cases were reported in India indicating potential enhancement of concurrent DENV infections. Therefore, accurate determination of the occurrence of DENV serotype coinfections needs to be conducted in various DENV prone parts of India. In this context, the present study was conducted to analyse the magnitude of concurrent infection in northern Kerala, a southwest state of India, during three consecutive years from 2013 to 2015. Methods: A total of 120 serum samples were collected from the suspected dengue patients. The serum samples were diagnosed for the presence of dengue NS1 antigen followed by the isolation of dengue genome from NS1 positive samples. The isolated dengue genome was further subjected to RTPCR based molecular serotyping. The phylogenetic tree was constructed based on the sequence of PCR amplified products. Results: Out of the total number of samples collected, 100 samples were positive for dengue specific antigen (NS1) and 26 of them contained the dengue genome. The RTPCR based molecular serotyping of the dengue genome revealed the presence of all four serotypes with different combinations. However, serotypes 1 and 3 were predominant combinations of concurrent infection. Interestingly, there were two samples with all four serotypes concurrently infected in year 2013. Discussion: All samples containing dengue genome showed the presence of more than one serotype, indicating $100 \%$ concurrent infection. However, the combination of serotypes 1 and 3 was predominant. To the best of our knowledge, this is the first report indicating the concurrent infection of dengue in the 
northern Kerala, India. The phylogenetic analysis of dengue serotype 1 identified in this study shows a close relationship with the strain isolated in Delhi and South Korea during the 2006 and 2015 epidemics respectively. Similarly this study indicates that the phylogeny of dengue serotype 3 of northern Kerala is more closely related to dengue isolate of Rajasthan state, India. The geographical and climatic conditions of Kerala favours the breeding of both the mosquito vectors of dengue (Aedes albopictus and Aedes aegypti). which may enhance the severity of dengue in the future. Therefore, the study provides an alarming message for the urgent need of an antiviral strategy or other health management systems to curb the spread of dengue infection. 
1 Occurrence of concurrent infections with multiple serotypes of dengue viruses during 2013

2 to 2015 in northern Kerala, India

3 Manchala Nageswar Reddy ${ }^{1}$, Ranjeet Dungdung ${ }^{1}$, Lathika Valliyott ${ }^{1}$, Rajendra

$4 \quad$ Pilankatta ${ }^{*}$

$5{ }^{1}$ Department of Biochemistry and Molecular Biology, School of Biological Sciences, Central

6 University of Kerala, India

$7 \quad *$ Correspondence Author:

8 Rajendra Pilankatta,

9 E-mail address: praj74@cukerala.ac.in. 


\section{Abstract}

26 Background: Dengue is a global human public health threat, causing severe morbidity and

27 mortality. The occurrence of sequential infection by more than one serotype of dengue virus

28 (DENV) is a major contributing factor for the induction of Dengue hemorrhagic fever [DHF] and

29 Dengue shock syndrome [DSS], two major medical conditions caused by DENV infection.

30 However, there is no specific drug or vaccine available against dengue infection. There are

31 reports indicating the increased incidence of concurrent infection of dengue in several tropical

32 and subtropical regions. Recently, increasing number of DHF and DSS cases were reported in

33 India indicating potential enhancement of concurrent DENV infections. Therefore, accurate

34 determination of the occurrence of DENV serotype co-infections needs to be conducted in

35 various DENV prone parts of India. In this context, the present study was conducted to analyse

36 the magnitude of concurrent infection in northern Kerala, a southwest state of India, during three

37 consecutive years from 2013 to 2015. Methods: A total of 120 serum samples were collected

38 from the suspected dengue patients. The serum samples were diagnosed for the presence of

39 dengue NS1 antigen followed by the isolation of dengue genome from NS1 positive samples.

40 The isolated dengue genome was further subjected to RTPCR based molecular serotyping. The

41 phylogenetic tree was constructed based on the sequence of PCR amplified

42 products. Results: Out of the total number of samples collected, 100 samples were positive for

43 dengue specific antigen (NS1) and 26 of them contained the dengue genome. The RTPCR based

44 molecular serotyping of the dengue genome revealed the presence of all four serotypes with

45 different combinations. However, serotypes 1 and 3 were predominant combinations of

46 concurrent infection. Interestingly, there were two samples with all four serotypes concurrently 
47 infected in year 2013. Discussion: All samples containing dengue genome showed the presence

48 of more than one serotype, indicating $100 \%$ concurrent infection. However, the combination of

49 serotypes 1 and 3 was predominant. To the best of our knowledge, this is the first report

50 indicating the concurrent infection of dengue in the northern Kerala, India. The phylogenetic

51 analysis of dengue serotype 1 identified in this study shows a close relationship with the strain

52 isolated in Delhi and South Korea during the 2006 and 2015 epidemics respectively. Similarly

53 this study indicates that the phylogeny of dengue serotype 3 of northern Kerala is more closely

54 related to dengue isolate of Rajasthan state, India. The geographical and climatic conditions of

55 Kerala favours the breeding of both the mosquito vectors of dengue (Aedes albopictus and Aedes

56 aegypti) which may enhance the severity of dengue in the future. Therefore, the study provides

57 an alarming message for the urgent need of an antiviral strategy or other health management

58 systems to curb the spread of dengue infection. 


\section{Introduction}

72 Dengue virus (DENV) belongs to the family of Flaviviridae and genus Flavivirus and poses a 73 global threat resulting in significant morbidity and mortality. The virus is transmitted by day-

74 biting mosquito, Aedes aegypti (Liu et al., 2014). However, there is no vaccine or antiviral drug 75 available that can neutralize all the four serotypes of dengue viruses.

76 There are four distinct DENV1-4 serotypes circulating all over the world and causing DENV 77 infection. The infection causes symptoms ranging from acute febrile illness to severe 78 manifestations, including bleeding and organ failure resulting in the DHF or DSS (Gubler et al., 79 1998; Moi et al., 2016). Co-infection with circulating DENV 1 and DENV 2 was reported in the 80 year 1982 in Columbia (Gubler et al., 1985). It has been known that sequential infection of more 81 than one serotype of dengue increases the severity of dengue symptoms (Hammon et al., 1973).

82 Meanwhile, there are reports indicating concurrent infection of dengue with more than one 83 serotype (Anoop et al., 2010 ). However the correlation between concurrent infection of dengue 84 with more than one serotype and severity of the disease symptoms is not well established. In this 85 context, the current study becomes highly relevant and gives a platform for future investigation 86 to understand the severity of the disease and concurrent infection caused by different dengue 87 serotypes. 
88 In the last 50 years, co- circulation of dengue serotypes was reported in South Asia, including

89 India. The first virologically confirmed dengue case was reported in the east coast of Calcutta,

90 India during 1963-64 (Carey et al., 1967; Sarkar et al., 1964). In addition, a dengue outbreak at

91 Kanpur, India was documented during the year 1968 by DENV 4 (Chaturvedi et al., 1970). The

92 presence of DENV 3 was found in patients as well as A.egypti mosquitoes in Vellore, India in

93 1966, and since then all the four types of DENV have been co circulated and isolated from

94 patients and mosquitoes (Myers et al., 1967; Wenming et al., 2005).

In 1996, DENV 2 serotype infections were noticed in India, followed by spreading all over the country (Shah et al., 2004; Singh et al., 2000). The capital city of India, Delhi, became a hyper endemic by hosting all four dengue virus serotypes by the year 2003 (Dar et al., 2003) with the coinfection of DENV 1 and DENV 3 in year 2005 (Gupta et al., 2006). The magnitude of concurrent infection (19\%) observed during the Delhi outbreak in 2006 is much higher in comparison with Taiwan (9.5\%) and Indonesia (11\%). Furthermore, replacement of DENV 2 and 3 with DENV 1 as the predominant serotype in Delhi over a period of three years (2007-2009) has been reported.

The occurrence of dengue fever was reported in the Kottayam district of Kerala, a south-western region of India, followed by an outbreak in year 2003. Concurrent infection with all three DENV 1-3 were reported in a large number of patients in year 2008 (Anoop et al., 2010) in Ernakulum district, a central region of Kerala. In year 2013, various parts of India, including Davangere and Central Karnataka out of 123 positive NS1 antigen samples 56(45.5\%) were infected with dengue fever, 37(30.1\%) with DHF, and 30(24.4\%) with DSS (Kalappanvar et al., 2013).

Since dengue cases are increasing at an alarming rate and causing a major health threat in tropical countries, it is necessary to identify and confirm the viral serotypes through 
111 epidemiological surveillance studies. The present study was conducted in order to specifically

112 identify dengue virus and to assess the concurrent infection in the northern Kerala (Malabar 113 region), India during three consecutive years (2013-2015). Some of these areas are ideal

114 ecosystems for the proliferation of Aedes albopictus mosquitoes. In this study, NS1 positive 115 samples were further tested by amplification of the junction region of capsid and pre membrane 116 gene (CprM) of the dengue viral genome (Lanciotti et al., 1992) by single step reverse 117 transcriptase polymerase chain reaction (RT-PCR) followed by specific serotype identification.

118 To the best of our knowledge, we are reporting for the first time the $100 \%$ concurrent infection 119 by different dengue serotypes among the samples analysed, including two samples which were 120 positive for all four dengue serotypes.

121

122

123

124

125

126

127

128

129

130

131

132

133 


\section{Patients and Methods}

144 Sample collection: The viremic blood samples from patients with suspected dengue symptoms 145 (as per WHO guidelines) were collected from the Government hospital and local diagnostic 146 centres located in the northern Kerala, India. The Institutional Ethics committee (IEC) clearance 147 (O.R.No:IAD/IEC/13/14) was taken from the Institute of Applied Dermatology (IAD), 148 Kasaragod district, Kerala for conducting this study. The prior informed consent was obtained 149 from all participating human subjects. The blood samples used in this study were collected 150 between June and August of year 2013, 2014, and 2015 respectively. The blood samples were 151 collected from patients who came to the diagnostic centres with a doctor's prescription or with 152 the suspicion (1-5days ). Donors were aged 5-60 years of either sex, including paediatrics (0-5 153 years old). Two millilitres of blood was collected in a vacutainer tube from each individual and 154 serum was separated by centrifugation as per the standard procedure.

155 Detection of NS1 antigen by capture ELISA: The presence of NS1antigen in the patient 156 serum sample was screened for using a DENV specific NS1 detection Enzyme Linked 
157 Immunosorbent Assay (ELISA) kit (Jmitra and Co. , New Delhi, India). The kit contains

158 monoclonal antibodies against NS1 coated on micro wells, which can detect NS1 antigen

159 secreted by DENV in the infected patient. Fifty microliters of serum sample per micro well was

160 used in the 96 well plate assay. Normal serum specimens obtained from healthy humans of same

161 sex and age groups were included as controls.

162 Isolation of dengue viral RNA: Dengue viral RNA was extracted from NS1 positive serum 163 samples using the pure link RNA mini kit (Invitrogen, USA) according to the manufacturer's 164 instructions. Clarified human serum sample of 150 microliters volume was used for RNA 165 isolation. All RNA samples were examined for their purity and concentration using Nano 166 photometer (Thermo scientific, 2000C).

167 Amplification of dengue viral CprM region by RT-PCR : Isolated Dengue viral RNA from 168 serum samples was subjected to single step reverse transcription polymerase chain reaction (RT169 PCR) (Lanciotti et al., 1992). Ten microliters of PCR amplified product was analysed on a 1\% 170 agarose gel and the size compared with a $1 \mathrm{~Kb}$ plus DNA ladder.

171 Nested PCR: A second round of amplification was initiated using one microliter of the above

172 PCR product (1:100 in sterile distilled water) as a template in the subsequent nested PCR

173 reaction. The reaction mixture contained all the components necessary for PCR amplification

174 including D1 as a forward primer and dengue virus type-specific reverse primers TS1, TS2, TS3, 175 and TS4 in separate individual tubes. The CprM consensus regions and each serotype-specific 176 primers were designed as mentioned by Lanciotti et al., 1992 with slight modification of TS4 177 primer

178 5'-CTCTGTTGTCTTAAACAAGAGA-3'. The PCR amplified products were analysed by 179 electrophoresis on 1\% agarose gel. 
180 Nucleotide sequencing: All necessary Good Lab Practices (GLP) were employed to avoid

181 artifacts. The precautions were taken to avoid the contamination of serum samples by bar coding

182 of samples. The cross contamination was prevented by setting of the PCR independently in the

183 separate tubes with pair of specific primers. The tube containing no template was also setup as a

184 negative control. The RT-PCR product (511 bp) obtained using D1 and D2 primers was

185 separated on the 1\% agarose gel. The DNA band was eluted from the gel using Wizard gel and

186 PCR clean up system (Promega, USA) as per the manufacturer instructions and was sequenced

187 directly using big dye terminator V3.1 ready reaction sequencing mixture in an automated

188 AB3500 Genetic Analyzer (Applied bio systems, Foster City, CA, USA). Additionally, nested

189 PCR products obtained using D1 and TS1/TS3 dengue virus serotype specific primers were

190 purified and sequenced as detailed above.

191 Amino Acid sequence similarity and diversity: The nucleotide sequences obtained from the 192 above method were submitted to GenBank database (NCBI). These sequences were translated 193 using the Expasy translation (EMBL) tool. These homologues amino acid regions were aligned 194 with the partial or full length amino acid sequences of dengue isolates of diverse geographical 195 locations (KP406801, EF127001, DQ285562, JQ922545,JN903581,KM403635, KR024707, 196 KT187563, KP723473, JQ917404, JN713897) retrieved from GenBank, using BioEdit sequence 197 alignment editor.

199 The phylogenetic analysis was conducted independently for DENV 1 (GenBank accession no. KJ954284) and DENV 3 (GenBank accession no. KM042094) of northern Kerala with the gene sequences of dengue virus isolates of different locations available in NCBI GenBank. The percentage of the bootstrap supporting values was shown at major nodes on the tree. The tree is 
203 drawn to scale, with branch lengths measuring the number of substitutions per site. The

204 reliability of the analysis was evaluated by a bootstrap test (MEGA),Tamura-Nei model (Tamura

205 et al., 2012). All positions containing gaps and missing data were eliminated.

206

207

208

209

210

211

\section{Results}

213 There were, in total, 120 serum samples of dengue infected individuals analysed between 2013

214 and 2015. Among these 120 samples, a total of 62 (51\%) were male, 35 (29\%) female, and 23

$215(20 \%)$ paediatric (0-5 years old) dengue cases (Figure 1). Both males and females belong to $30-$

21640 years age group with an approximate ratio of $2: 1$. This ratio is similar with the previous

217 reported value (Mishra et al., 2015). There were 23 (20\%) pediatric (0-5 years old) cases,

218 consisting of 14 males and 9 females. Out of 120 serum samples, $100(83 \%)$ were found to be

219 NS1 positive, indicating the possibility of finding dengue viral RNA genome.

220 Dengue viral genome isolation and serotyping: Dengue viral RNA was isolated from NS1

221 positive serum samples, as detailed in the patients and methods section. The isolated RNA was

222 subjected to RT-PCR based amplification (Figure 2) using D1 and D2 primers. There was

223 dengue specific amplified product of size 511 bp present only in 26 samples. This observation 
224 confirms the presence of dengue genome in these 26 samples out of 100 samples, which were

225 NS1 positive. The initial dengue NS1 antigen detection and the presence of identifying dengue

226 genome in the serum samples indicates the possibility of dengue infection. A representative gel

227 image, which indicates the presence of $511 \mathrm{bp}$ amplified product is given in Figure 3A. Out of

228 fifty-one NS1 positive samples in the year 2013, thirteen samples were found to be positive for

229 dengue genome. Similarly, there were three out of seventeen and ten out of thirty two samples

230 positive for dengue genome analysed during 2014 and 2015 respectively (Table I). All samples

231 were co-infected with more than one serotype with various combinations of serotypes as given in

232 Table I and Figure 3 (B, C, D).

233 Concurrent infection: The above amplified RTPCR product (511 bp) was used as a template for

234 nested PCR, using serotype specific primers as detailed in methods section. The nested PCR

235 analysis of dengue serotyping showed that a single individual is hosting for more than one

236 dengue virus serotype circulating in the blood. In all 26 samples of multiple infection, three

237 different serotypes co-existed in eight samples, two different serotypes co- existed in sixteen

238 samples, and four different serotypes co-existed in two samples. There are nine cases of

239 concurrent infection with DENV1 and DENV 3, seven cases with DENV 2 and DENV 3, eight

240 cases with DENV 1, 2 and 3, and two cases with DENV 1, 2, 3 and 4 combinations as shown in

241 the Table I. There was a large number of concurrent infection with DENV 1 and DENV 3. The

242 concurrent infection of all four serotypes is an alarming indication and needs to be investigated

243 in detail. The sequential infection with more than one serotype of a particular region leads to a

244 severe cause for eliciting antibody dependent immune response.

245 Nucleotide sequencing and dengue virus serotyping : The D1 and D2 primer based amplified

246 PCR product corresponding to CPrM region was sequenced (GenBank accession no. 
247 KX031992) and the data confirmed the presence of dengue viral genome sequence in the

248 samples. The nucleotide sequence was found to be $99 \%$ similar with the existing CprM region of

249 DENV 3 viral strains in GenBank database. Blast analysis, using CprM junction nucleotide

250 sequence, showed similarity with DENV 3 isolates from Pakistan with 99\% query coverage

251 (GenBank accession no.KF041254). Nucleotide sequencing of DENV 1 specific nested PCR

252 product, obtained using D1 and TS1 primer combinations, gives the size of 423 bp (GenBank

253 accession no. KJ954284). The corresponding amino acid sequence (1-139 aa) derived from

254 DEV 1 specific amplified region represents non functional poly protein partial sequence. DENV

2553 specific nested PCR product using D1 and TS3 primer combinations gives the size of $235 \mathrm{bp}$

256 (GenBank accession no. KM042094) on a $1 \%$ agarose gel. However, PCR amplified product of

257 DENV 2 and DENV 4 were the lesser in quantity, the nucleotide sequences were not obtained

258 with clarity.

259 Amino Acid sequence diversity: To observe any mutations, the amino acid sequence of

260 DENV1 was compared with the other closely related DENV 1 strains. The comparison of the

261 amino acid sequence (1-139 aa) of DENV 1 (CUKKEL201308001; GenBank accession no.

262 KJ954284) with various DENV 1 isolates existing in the data base (GenBank accession no

263 KP406801, EF127001, DQ285562,JQ922545,JN903581,KM403635, KR024707, KT187563,

264 KP723473, JQ917404, JN713897) revealed an identity of 98\%-99\%, except for Valine (V)

265 which is replaced by Isoleucine (I) at 131st position as shown in Figure 4. However,

266 replacement of Isoleucine with Valine was reported at residue 106 of the capsid protein in the

267 isolates obtained from the 1997, 1998 and 2001 outbreaks in the Caribbean islands pertaining to

268 the Asian/American genotype (Gardella et al., 2008). 
269 Phylogenetic analysis of DENV 1 and DENV 3: In the molecular phylogenetic analysis (Figure

270 5) by Maximum Likelihood method, the bootstrap support values indicates that the sequence of

271

272

273 274 for the analysis (Figure 5). Similarly the phylogenetic analysis (Figure 6) of dengue 3 virus

275 isolate of Northern Kerala (CUKKEL2013/02; GenBank accession no. KM042094) indicates

276

277

278

279

280

281

282

283

284

285

286

287

288

289

290

291 that this strain is closely related to Dengue virus isolate (DENV-3/DMRC/Bal87/2013)

polyprotein gene, partial cds (KT239735) of Rajasthan, India.

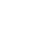
ancestor relationship with DENV 1 isolate (GenBank accession no. KP406801) and DENV 1 isolate 06/1/del2006 (GenBank accession no. EF127001) among 28 nucleotide sequences used (CUKKEL2013/02; GenBank accession no. KM042094) indicates 
292 was observed in Delhi in 2006 (Bharaj et al., 2008). In this outbreak, 9 out of 48 samples (19\%)

293 were identified positive for dengue virus as a concurrent infection with more than one dengue

294 virus serotype. The concurrent infection with the involvement of three dengue viral serotypes

295 (DENV 1, 2, and 3) at an approximate rate of 56.8\% was observed in Ernakulam, Kerala state in

2962008 (Anoop et al., 2010). A large outbreak of dengue fever (DF) was reported in 2007, in the

297 locality of Indo-Myanmar boarder with the co circulation of concurrent infection of DENV 2 \&

$2983,1 \& 3$, and $1 \& 4$ serotypes (Khan et al., 2013).

299 In the year 1980, DENV 1 and 2 were first observed in Columbia. By 1994, many parts of the 300 world, including, Bolivia, Brazil, Costa Rica, El Salvador, French Guiana, Guatemala, 301 Honduras, Mexico, Nicaragua, Panama, Peru, Puerto Rico, Trinidad and Tobago, and Venezuela 302 were hosting all four dengue serotypes (Loron et al., 1999). The first case of a dual infection with 303 two dengue virus serotypes (DENV 1 and DENV 4) was reported in the serum of a 16 year old 304 male during the 1982 outbreak in Puerto Rico (Gubler et al., 1985). Whereas in New Caledonia, 305 DENV 1 and DENV 3 viruses were isolated from 6 patients with Dengue fever in 1989 (Laille et 306 al., 1991). The first report of dual concurrent infection with DENV 2 and DENV 3 was observed 307 in Chinese patients returned from Sri Lanka (Peng et al., 2005). The viremic serum samples 308 (292 in total) collected during epidemics from Indonesia, Mexico, and Puerto Rico were tested, 309 and $16(5.5 \%)$ cases were found to contain two or more dengue viruses by reverse transcriptase310 polymerase chain reaction (Loron et al., 1999).

311 Out of 100 NS1 positive samples, 26 (26\%) were found to be containing dengue viral RNA and 312 showed a target specific band $(511 \mathrm{bp})$ on an agarose gel. The low proportion of dengue viral 313 genome in NS1 positive serum samples may be due to loss of intact viral genome or the 314 degradation of dengue positive RNA sense strand while handling the samples. The serotyping of 
315 the dengue viral RNA samples using nested PCR with sero specific primers revealed that all

316 samples are concurrently infected with multiple serotypes. Different combinations of Dengue

317 virus (DENV) concurrent infections including DENV 1 and DENV 3, 34\% (9 cases out of 26

318 cases ), DENV 2 and DENV 3, 27\% ( 7 cases out of 26 cases), DENV 1,2 and 3, 31\% (8 cases

319 out of 26 cases) were observed. Further, it was also observed that two of the samples were

320 concurrently infected with all four serotypes as shown in Table 1 . However, coexistence of all

321 four serotypes in one host needs to be authenticated by characterizing the viral genome after

322 isolation and propagation of viruses from those serum samples. The observations indicate the

323 possibility of enhancement of future concurrent infections, as the percentage of single serotype

324 infection decreased and concurrent infection of multiple serotypes increased based on the current

325 investigation. As supporting evidence, there were only $19 \%$ of concurrent infections during

3262006 (Delhi, India). However, there was a significant increase in the percentage of concurrent

327 infections in 2009 (56.8\%) in Ernakulum, India. The percentage of concurrent infection reached

$328100 \%$ based on the current investigation. It is also important to note that Kerala, a southwest

329 state of India, provides an ideal ecosystem for the propagation of both the mosquito vectors

330 (Aedes albopictus and Aedes aegypti) of dengue transmission.

331 Since the maximum nucleotide sequence was obtained in the case of DENV 1, the amino acid

332 alignment (1-139 aa) was made with the various DENV 1 isolates existing in the data base,

333 which revealed an identity of $98 \%-99 \%$, except Isoleucine instead of Valine at $131^{\text {st }}$ position as

334 shown in Figure 4. Since both amino acids belong to the non polar group of standard genetic

335 code, the amino acid change may not have any significance. Phylogenetic analysis of DENV 1

336 nucleotide sequences (GenBank accession no. KJ954284) obtained from 2013 epidemic samples

337 shares a common ancestor relationship with clinical isolates of DENV 1 from South Korean 
338 Travellers (GenBank accession no. KP406801) as well as the DENV 1 isolate from Delhi, India

339 (GenBank accession no. EF127001) in among 28 nucleotide sequences used for the analysis

340 (Figure 5). Similarly the phylogenetic analysis (Figure 6 ) of dengue 3 virus isolate of northern

341 Kerala indicates the close relationship to Dengue virus isolate (DENV-3/DMRC/Bal87/2013)

342 polyprotein gene, partial cds (KT239735) of Rajasthan, India. Concurrent infection of multiple

343 serotypes of dengue poses a lot of questions regarding viral replication. The possibility of mutual

344 interference among different dengue serotypes during replication within the same host need to be

345 investigated in detail. If so, would there be any replicative advantage to one of the serotypes

346 against the other three serotypes? The viral interference which leads to replicative advantage

347 towards one particular serotype is a major concern in the development of tetravalent dengue

348 vaccine (Anderson et al., 2011).

\section{Conclusions:}

350 The present study shows that all samples which were able to show PCR amplified product,

351 analysed from the northern Kerala, India between 2013 and 2015 harbour more than one

352 serotype of dengue, indicating $100 \%$ concurrent infection. The occurrence of concurrent

353 infection of DENV 1 and DENV 3 was higher as compared to other combinations. The DENV 1

354 isolates of Northern Kerala was more closely related to South Korean and Delhi strain based on

355 phylogenetic analysis. Similarly DENV 3 was more closely related to Dengue 3 isolates of

356 Rajasthan, India ((KT239735). 
359 We thank Mr. Sabastian, Director at the division of Kanhangad diagnostic centre for providing 360 the clinical samples for research purposes. We also thank all the medical staff and administrative 361 staff of IAD (Institute of Applied Dermatology), Uliyathadka, Kasargod District, Kerala who 362 have kindly assisted in sample processing.

\section{References}

1. Alcon S, Talarmin A, Debruyne M, Falconar A, Deubel V, Flamand M.( 2002) Enzymelinked immunosorbent assay specific to Dengue virus type 1 nonstructural protein NS1 
reveals circulation of the antigen in the blood during the acute phase of disease in patients experiencing primary or secondary infections. J Clin Microbiol 40, 376-81.

2. Anderson KB, Gibbons RV, Edelman R, Eckels KH, Putnak RJ, Innis BL, Sun W. (2011) Interference and facilitation between dengue serotypes in a tetravalent live dengue virus vaccine candidate. J Infect Dis 204, 442-50.

3. Anoop M, Issac A, Mathew T, Philip S, Kareem NA, Unnikrishnan R, Sreekumar E. (2010) Genetic characterization of dengue virus serotypes causing concurrent infection in an outbreak in Ernakulam, Kerala, South India. Indian J Exp Biol 48, 849-57.

4. Bharaj P, Chahar HS, Pandey A, Diddi K, Dar L, Guleria R, Kabra SK, Broor S. (2008) Concurrent infections by all four dengue virus serotypes during an outbreak of dengue in 2006 in Delhi, India. Virol J 9, 5:1.

5. Chaturvedi UC, Mathur A, Kapoor AK, Mehrotra NK, Mehrotra RM. (1970) Virological study of an epidemic of febrile illness with haemorrhagic manifestations at Kanpur, India, during 1968. Bull World Health Organ 43, 289-93.

6. Dar L, Gupta E, Narang P, Broor S. (2003) Co-circulation of dengue serotypes, Delhi, India, 2003. Letter to the editor. Emerg Infect Dis 12, 352-353.

7. Deubel V, Laille M, Hugnot JP, Chungue E, Guesdon JL, Drouet MT, Bassot S,Chevrier D. (1990) Identification of dengue sequences by genomic amplification: rapid diagnosis of dengue virus serotypes in peripheral blood. J Virol Methods 30, 41-54.

8. Fatima Z, Idrees M, Bajwa MA, Tahir Z, Ullah O, Zia MQ, Hussain A, Akram M,Khubaib B, Afzal S, Munir S, Saleem S, Rauff B, Badar S, Naudhani M, Butt S,Aftab M, Ali L, Ali M. (2011).Serotype and genotype analysis of dengue virus by sequencing 
followed by phylogenetic analysis using samples from three mini outbreaks-2007-2009 in Pakistan. BMC Microbiol.10;11:200.

9. Gardella-Garcia CE, Perez-Ramirez G, Navarrete-Espinosa J, Cisneros A, JimenezRojas F, Ramírez-Palacios LR, Rosado-Leon R, Camacho-Nuez M, Munoz Mde L. (2008) Specific genetic markers for detecting subtypes of dengue virus serotype-2 in isolates from the states of Oaxaca and Veracruz, Mexico. BMC Microbiol 15, 8:117.

10. Gubler DJ, and Clark, G. G. (1995) Dengue/dengue hemorrhagic fever: the emergence of a global health problem. Emerg Infect Dis 1, 55-7.

11. Gubler DJ, Kuno G, Sather GE, Waterman SH. (1985) A case of natural concurrent human infection with two dengue viruses. Am Trop Med Hyg 34, 170-173.

12. Gubler DJ, Trent DW. (1994) Emergence of epidemic dengue/ dengue hemorrhagic fever as a public health problem in the Americas. Infect Agents Dis 2, 383-393.

13. Gubler DJ. (1998) Dengue and Dengue hemorrhagic fever. Clin Microbiol, Rev 11:480496.

14. Gupta E, Dar L, Kapoor G, Broor S. (2006) The changing epidemiology of dengue in Delhi, India. Virology Journal 3:92.

15. Holmes EC, Worobey M, Rambaut A. (1999) Phylogenetic evidence for recombination in dengue virus. Mol Biol Evol 16, 405-409.

16. I.M. Rocco, M.L. Barbosa, E.H. Kanomata. (1998) Simultaneous infection with dengue 1 and 2 in a Brazilian patient Rev. Inst. Med. Trop. Sao Paulo 40, 151-154.

17. Kalappanvar NK, VinodKumar CS, Basavarajappa KG, Chandrasekhar G, Sanjay D. (2013) Outbreak of dengue infection in rural Davangere, Karnataka. Asian Pac J Trop $\operatorname{Med}$ 6, 502-3. 
18. Khan SA, Dutta P, Borah J, Chowdhury P, Doloi PK, Mahanta J.(2013). Dengue outbreak in an Indo-Myanmar boarder area: epidemiological aspects and risk factors. Trop Biomed.30(3):451-8.

19. Lanciotti RS, Calisher CH, Gubler DJ, Chang GJ, Vorndam AV. (1992) Rapid Detection and typing of dengue viruses from clinical samples by using reverse transcriptase polymerase chain reaction. J Clin Microbiol 30, 545-51.

20. Leo YS, Thein TL, Fisher DA, Low JG, Oh HM, Narayanan RL, Gan VC, Lee VJ, Lye DC. (2011). Confirmed adult dengue deaths in Singapore: 5-year multi-center retrospective study. BMC Infect Dis. 12;11:123.

21. Liu-Helmersson J, Stenlund H, Wilder-Smith A, Rocklöv J. (2014) Vectorial capacity of Aedes aegypti: effects of temperature and implications for global dengue epidemic potential. PLoS One 9(3):e89783.

22. M. Laille, V. Deubel, F.F. (1991) Sainte-Marie Demonstration of concurrent dengue 1 and dengue 3 infection in six patients by the polymerase chain reaction. J. Med. Virol 34, $51-54$.

23. Mason PW, McAda PC, Mason TL, Fournier MJ. (1987) Sequence of the dengue-1 virus genome in the region encoding the three structural proteins and the major non structural protein NS1. Virology 161, 262-7.

24. Mishra G, Jain A, Prakash O, Prakash S, Kumar R, Garg RK, Pandey N, Singh M. (2015) Molecular characterization of dengue viruses circulating during 2009-2012 in Uttar Pradesh, India. J Med Virol 87, 68-75.

25. Moi ML, Takasaki T, Kurane I. (2016) Human antibody response to dengue virus: implications for dengue vaccine design. Trop Med Health 14, 44:1. 
26. Osatomi K, Sumiyoshi H. (1990) Complete nucleotide sequence of dengue type 3 virus genome RNA. Virology 176, 643-7.

27. Sarkar JK, Pavri KM, Chatterjee SN, Chakravarty SK, Aanderson CR. (1964)

Virological and serological studies of cases of haemorrhagic fever in Calcutta. Indian J Med Res 52, 684-691.

28. Shah I, Deshpande GC, Tardeja PN. (2004) Outbreak of dengue in Mumbai and predictive markers for dengue shock syndrome. J Trop Pediatr 50, 301-5.

29. Singh J, Balakrishnan N, Bhardwaj M, Amuthadevi P, George EG, Subramani K, Soundararajan K, Appavoo NC, Jain DC, Ichhpujani RL, Bhatia R, Sokhey J. (2000) Silent spread of dengue and dengue haemorrhagic fever to Coimbatore and Erode districts in Tamil Nadu, India, 1998: need for effective surveillance to monitor and control the disease. Epidemiol Infect 125, 195-200.

30. Tamura K., Battistuzzi FU, Billing-Ross P, Murillo O, Filipski A, and Kumar S. (2012) Estimating Divergence Times in Large Molecular Phylogenies. Proceedings of the National Academy of Sciences 109, 19333-19338.

31. Tamura K., Stecher G., Peterson D., Filipski A., and Kumar S. (2013) MEGA6: Molecular Evolutionary Genetics Analysis version 6.0. Molecular Biology and Evolution 30, 2725-2729.

32. W.M.Hammon. (1973) Dengue hemorrhagic fever--do we know its cause? Am J Trop Med Hyg. 22(1):82-91. Review

33. Wenming P, Man Y, Baochang F, Yongqiang D, Tao J, Hongyuan D, Ede Q. (2005) Simultaneous infection with dengue 2 and 3 viruses in a Chinese patient return from Sri Lanka. J Clin Virol 32, 194-198. 
34. Worobey M, Rambaut A, Holmes EC. (1999). Widespread intra serotype recombination in natural populations of dengue virus. Proc Natl Acad Sci USA 96:7352-7357.

35. Zhao B, Mackow E, Buckler-White A, Markoff L, Chanock RM, Lai CJ, Makino Y. (1986) Cloning full-length dengue type 4 viral DNA sequences: analysis of genes coding for structural proteins. Virology 155, 77-88. 
Figure 1

Bar diagram showing the number of dengue virus infected cases under different sex group in three consecutive years (2013-2015).

$X$ - axis represents year of study and $Y$ - axis represents number of dengue infected cases. A: Total number of dengue infected individuals. B, C, D: Dengue NS1 positive male, female and pediatrics respectively.

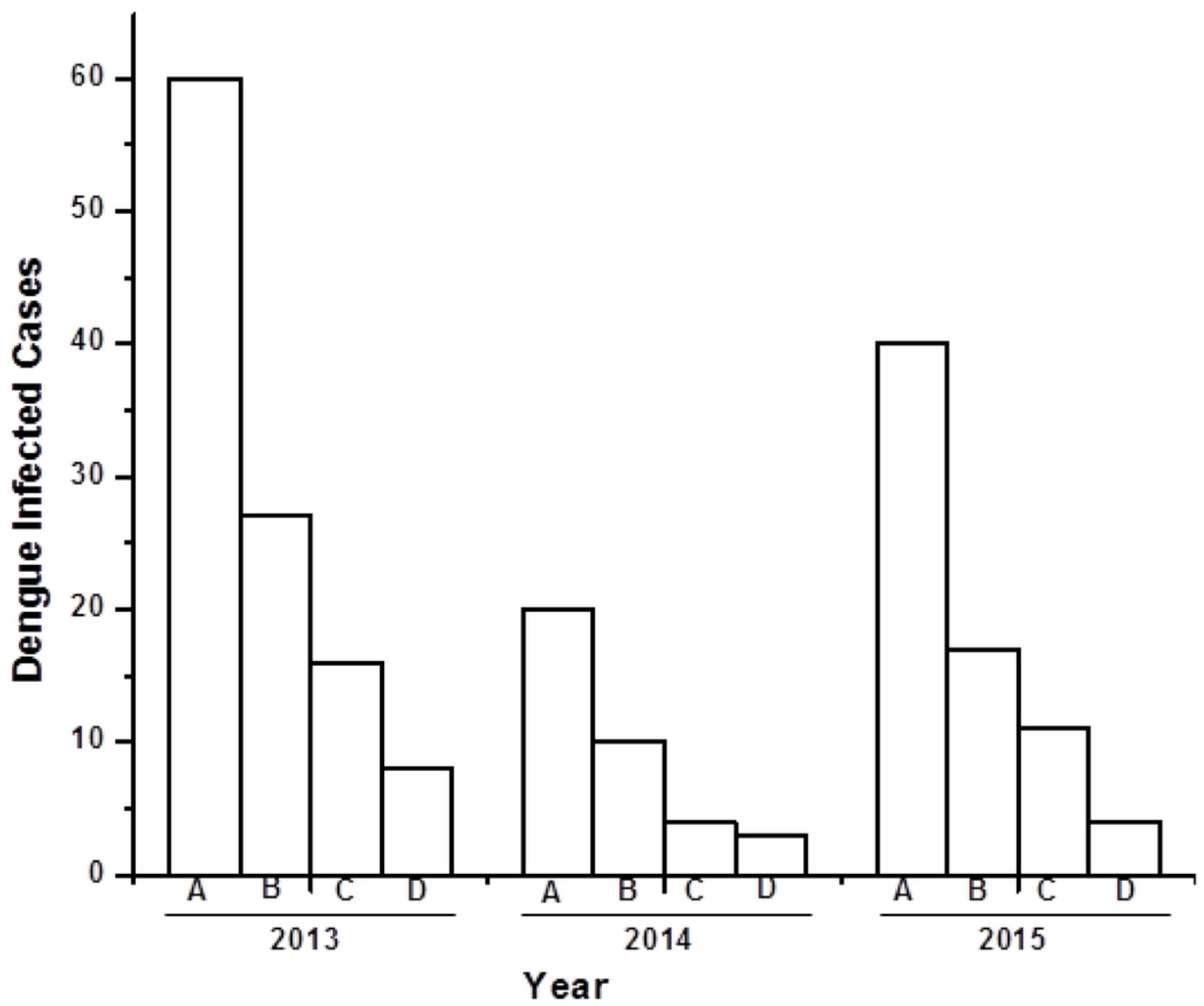


Figure 2

Schematic representation of dengue viral CprM junction amplification by RT-PCR.

A) Dengue viral RNA has 5' UTR region, three structural (capsid (C), pre membrane (prM), and envelope (E)) and seven nonstructural (NS1, NS2A, NS2B, NS3, NS4A, NS4B, and NS5) genes ends with 3' UTR region. B) CPrM junction (511 bp) of structural region was amplified with D1 and D2 primers using dengue viral RNA as a template. C) The amplified products with D1 and sero specific TS1, TS2, TS3 and TS4 primers independently, indicates the expected size (bp) 482, 119, 290 and 392 respectively by nested PCR.

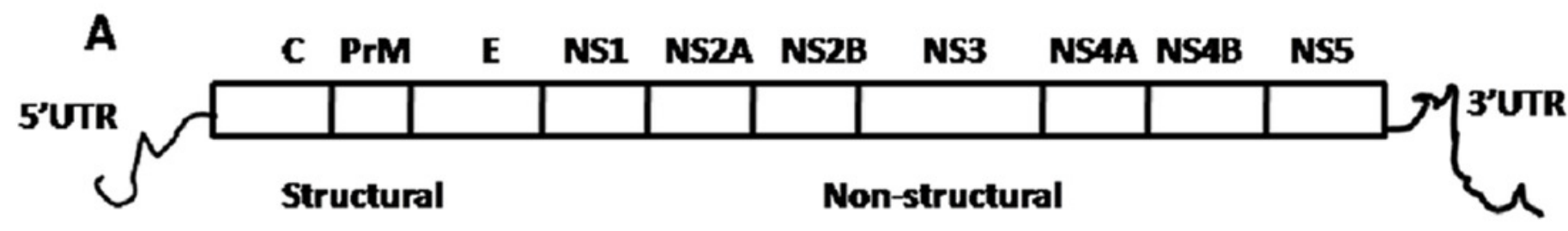

B
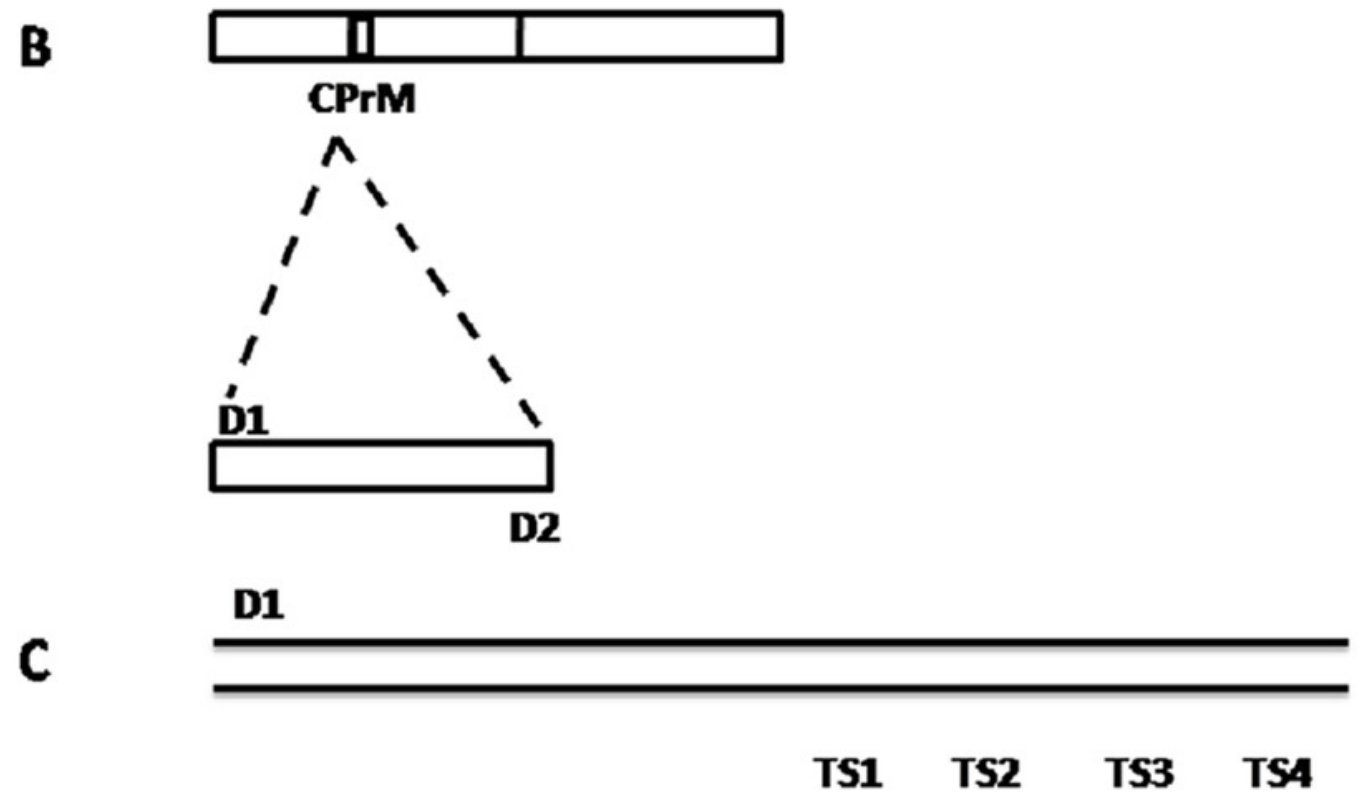


\section{Figure 3}

The agarose gel images (1\%) showing the amplified DNA from dengue viral genome using RT-PCR.

Figure A shows a representative DNA gel showing the 511 bp DNA fragment obtained by RTPCR using dengue viral RNA as template with D1 (forward) and D2 (reverse) primers. Lane 1 and Lane 4 shows the presence of dengue viral genome in the sample as evidenced by a clear DNA band of 511 bp size. However, Lane 2 and 3 did not show the presence of dengue viral genome in the corresponding samples. Figure $B, C$ and $D$ represents DNA gel images showing the nested PCR products by serotype specific primers with D1\&TS1 (Lane 1), D1\&TS2 (Lane 2), D1\&TS3 (Lane 3), D1\&TS4 (Lane 4)primer combinations using 511 bp PCR product as a template. In Figure B, Lane 1 and 4 shows the presence of amplified product of size $482 \mathrm{bp}$ and $392 \mathrm{bp}$ respectively indicating the presence of DEV 1 and 4 in the same individual. Similarly, Figure C Shows the presence amplified product of size 482 bp, 119 bp and $290 \mathrm{bp}$ in Lane 1, 2 and 3 respectively, indicating the concurrent infection of dengue serotype 1, 2 and 3 in the same individual. Interestingly, Figure $D$ indicates the concurrent infection of all four serotypes in the same individual as evidenced by the amplified product of size 482 bp, 119 bp 290 bp and 392 bp in all four lanes respectively. M, indicates 1kb DNA ladder (ladder size is shown on the left hand side of Figure A, and lane number indicates on the top of each figure). 


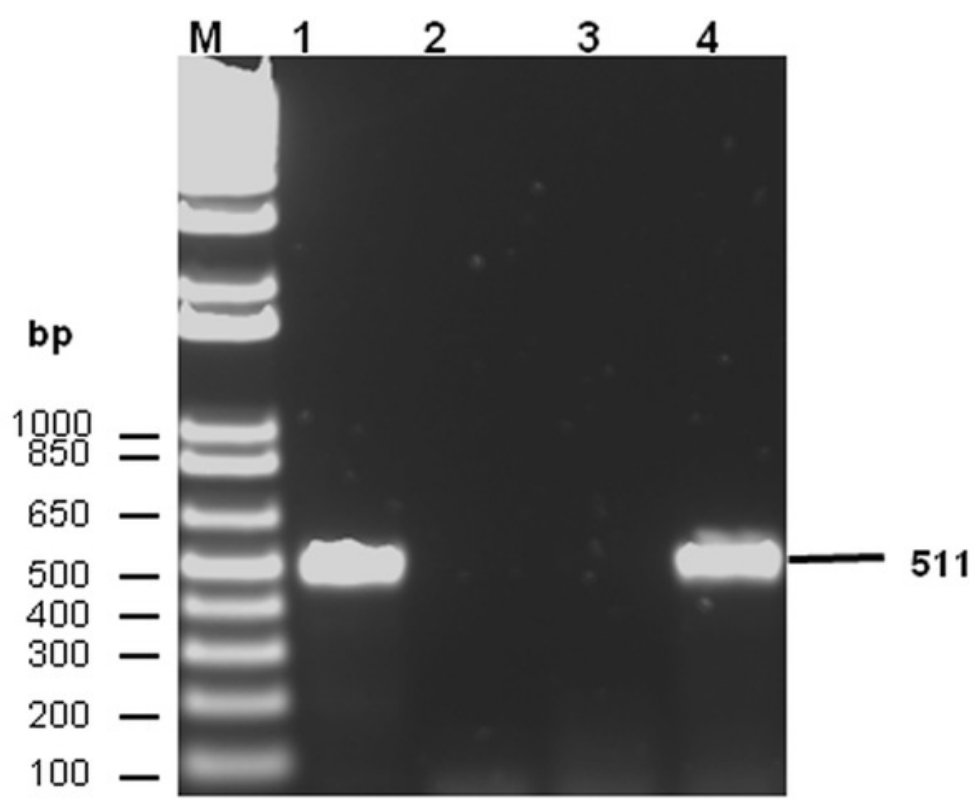

(A)

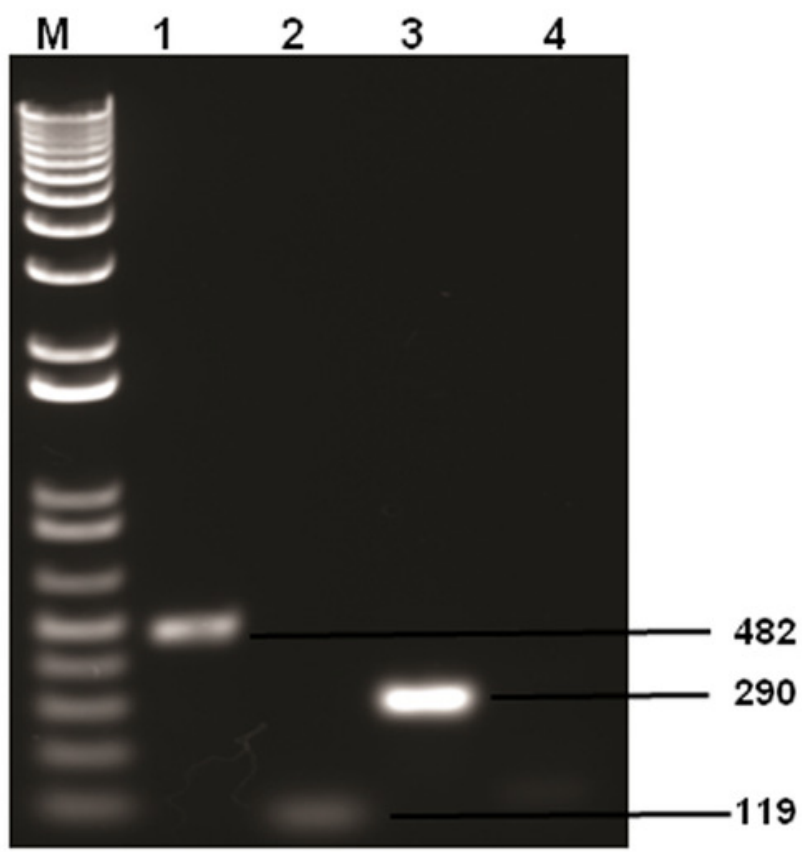

(C)

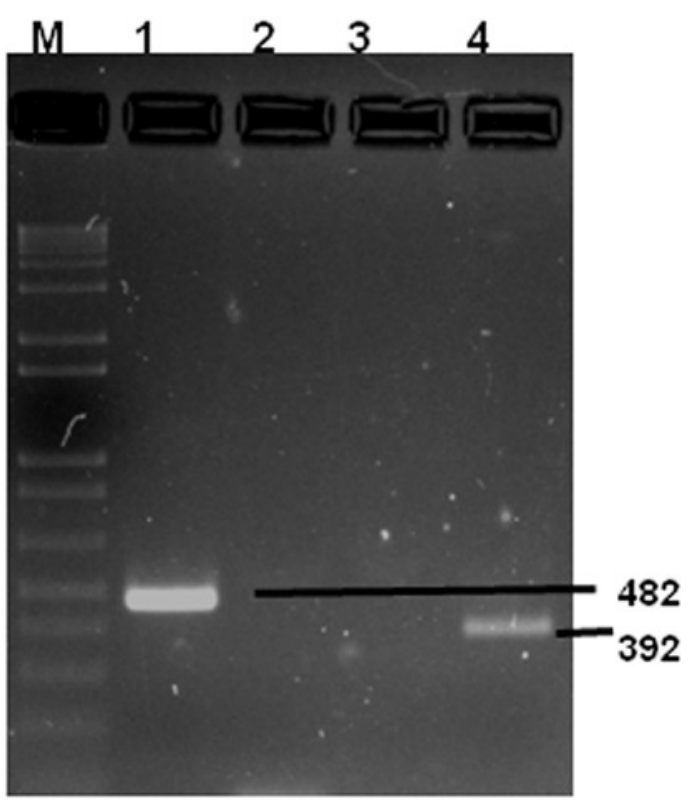

(B)

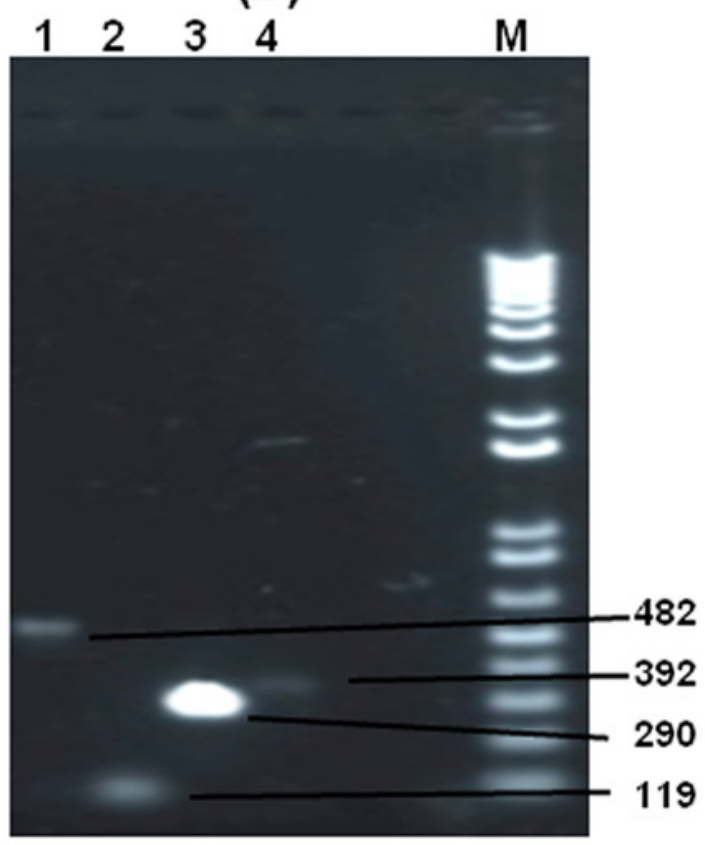

(D) 


\section{Figure 4}

Alignment of deduced amino acid sequence of Dengue serotype 1 (DENV 1).

The Amino acid sequence derived from DENV 1 specific amplified region (GenBank accession no.KJ954284) containing 139 amino acid residues were aligned with the other eleven DENV 1 amino acid sequences (GenBank accession no.KP406801, EF127001, DQ285562, JQ922545,JN903581,KM403635, KR024707, KT187563, KP723473, JQ917404, JN713897) of various strains retrieved from GenBank.The gene accession number of individual isolate are shown on the left. The alignment was edited with BioEdit sequence alignment editor. A mutation was observed in the test sample, where Valine was replaced with Isoleucine at $131^{\text {st }}$ position and it was highlighted in the image. 
KJ954284 KP4 06801 EF127001 DQ2 85562 JQ9 22545 JN9 03581 KM4 03635 KR024707 KT187563 KP7 23473 JQ9 17404 JN7 13897

KJ954284 KP4 06801 EF127001 DQ2 85562 JQ9 22545 JN9 03581 KM4 03635 KR024707 KT187563 KP723473 JQ9 17404 JN7 13897

KJ954284 KP4 06801 EF127001 DQ2 85562 JQ9 22545 JN9 03581 KM4 03635 KR024707 KT187563 KP723473 JQ9 17404 JN7 13897 $\ldots|\ldots|_{10} \ldots|\ldots|_{20} \ldots|\ldots|_{30} \ldots|\cdots|_{40} \ldots|\cdots|_{50}$

MLKRARNRVS TGSQLAKRFS KGLLSGQGPM KLVMAFIAFL RFLAIPPTAG MLKRARNRVS TGSQLAKRFS KGLLSGQGPM KLVMAFIAFL RFLAIPPTAG MLKRARNRVS TGSQLAKRFS KGLLSGQGPM KLVMAFIAFL RFLAIPPTAG MLKRARNRVS TGSQLAKRFS KGLLSGQGPM KLVMAFIAFL RFLAIPPTAG MLKRARNRVS TGSQLAKRFS KGLLSGQGPM KLVMAFIAFL RFLAIPPTAG MLKRARNRVS TGSQLAKRFS KGLLSGQGPM KMVMAFIAFL RFLAIPPTAG MLKRARNRVS TGSQLAKRFS KGLLSGQGPM KMVMAFIAFL RFLAIPPTAG MLKRARNRVS TGSQLAKRFS KGLLSGQGPM KMVMAFIAFL RFLAIPPTAG MLKRARNRVS TGSQLAKRFS KGLLSGQGPM KMVMAFIAFL RFLAIPPTAG MLKRARNRVS TGSQLAKRFS KGLLSGQGPM KMVMAFIAFL RFLAIPPTAG MLKRARNRVS TGSQLAKRFS KGLLSGQGPM KMVMAFIAFL RFLAIPPTAG MLKRARNRVS TGSQLAKRFS KGLLSGQGPM KLVMAFIAFL RFLAIPPTAG

$\ldots|\ldots|_{60} \ldots|\ldots|_{70} \ldots|\ldots|_{80} \ldots|\ldots|_{90} \ldots|\ldots|_{100}$ ILARWSSFKK NGAIKVLRGF KKEISSMLNI MNRRKRSVTM LLMLLPTALA ILARWSSFKK NGAIKVLRGF KKEISSMLNI MNRRKRSVTM LLMLLPTALA ILARWSSFKK NGAIKVLRGF KKEISSMLNI MNRRKRSVTM LLMLLPTALA ILARWSSFKK NGAIKVLRGF KKEISSMLNI MNRRKRSVTM LLMLLPTALA ILARWSSFKK NGAIKVLRGF KKEISSMLNI MNRRKRSVTM LLMLLPTALA ILARWSSFKK NGAIKVLRGF KKEISSMLNI MNRRKRSVTM LLMLLPTALA ILARWSSFKK NGAIKVLRGF KKEISSMLNI MNRRKRSVTM LLMLLPTALA ILARWSSFKK NGAIKVLRGF KKEISSMLNI MNRRKRSVTM LLMLLPTALA ILARWSSFKK NGAIKVLRGF KKEISSMLNI MNRRKRSVTM LLMLLPTALA ILARWSSFKK NGAIKVLRGF KKEISSMLNI MNRRKRSVTM LLMLLPTALA ILARWSSFKK NGAIKVLRGF KKEISSMLNI MNRRKRSVTM LLMLLPTALA ILARWSSFKK NGAIKVLRGF KKEISSMLNI MNRRKRSVTM LLMLLPTALA

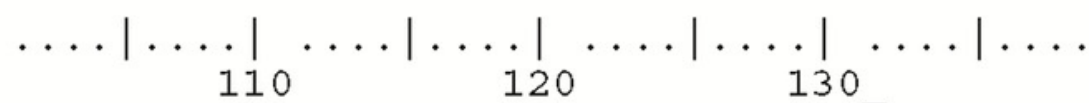
FHLTTRGGEP HMIVSKQERG KSLLFKTSAG INMCTLIAM FHLTTRGGEP HMIVSKQERG KSLLFKTSAG MNMCTLIAM FHLTTRGGEP HMIVSKQERG KSLLFKTSAG NNMCTLIAM FHLTTRGGEP HMIVSKQERG KSLLFKTSAG VNMCTLIAM FHLTTRGGEP HMIVSKQERG KSLLFKTSAG NNMCTLIAM FHLTTRGGEP HMIVSKQERG KSLLFKTSAG NNMCTLIAM FHLTTRGGEP HMIVSKQERG KSLLFKTSAG NNMCTLIAM FHLTTRGGEP HMIVSKQERG KSLLFKTSAG NNMCTLIAM FHLTTRGGEP HMIVSKQERG KSLLFKTSAG VNMCTLIAM FHLTTRGGEP HMIVSKQERG KSLLFKTSAG NNMCTLIAM FHLTTRGGEP HMIVSKQERG KSLLFKTSAG VNMCTLIAM FHLTTRGGEP HMIVSKQERG KSLLFKTSAG VNMCTLIAM 


\section{Figure 5}

Molecular phylogenetic analysis of DENV 1 by Maximum Likelihood method, Tamura-Nei model.

The phylogenetic tree was constructed from the gene sequences of DENV isolates of different geographical locations and DENV 1 isolate (GenBank accession no.KJ954284) of northern Kerala. The bootstrap support value indicates that the sequence of dengue virus isolate from Northern Kerala shares a close relationship with DENV 1 isolate (GenBank accession no.KP406801) and DENV 1 isolate 06/1/del2006 (GenBank accession no.EF127001). The reliability of the analysis was evaluated by a bootstrap test with 1,000 replications (MEGA 6). The analysis involved total 28 nucleotide sequences.

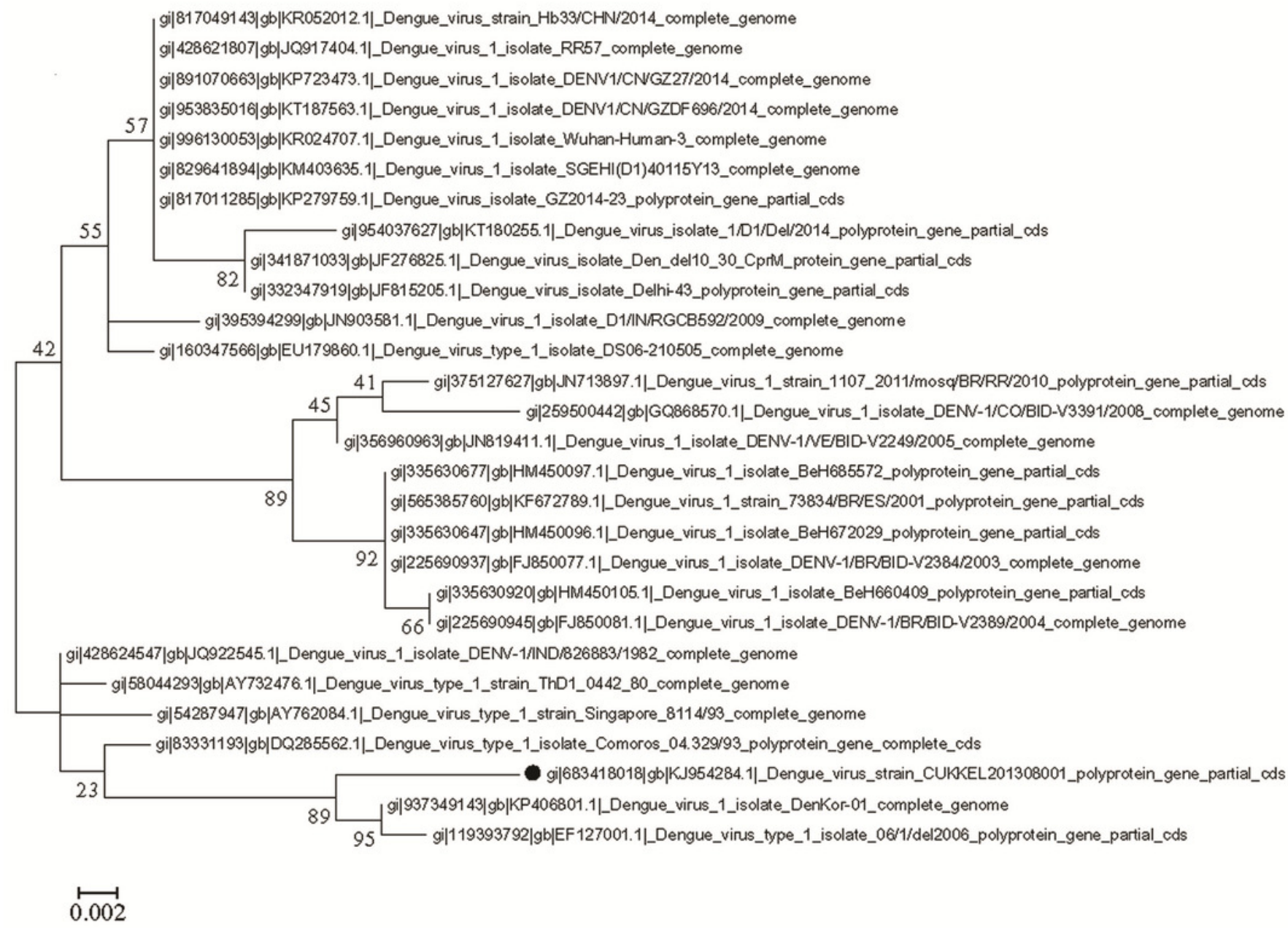




\section{Figure 6}

Molecular phylogenetic analysis of DENV 3 by Maximum Likelihood method, Tamura-Nei model.

The phylogenetic tree was constructed based on dengue gene sequences of different geographical isolates and DENV 3 isolate obtained from northern Kerala (CUKKEL2013/02; GenBank accession no. KM042094). The analysis indicates that northern Keralian isolate is closely related to Dengue virus isolate (DENV-3/DMRC/Bal87/2013) polyprotein gene, partial cds (KT239735) of Rajasthan, India. The reliability of the analysis was evaluated by a bootstrap test with 500 replications (MEGA 7),Tamura-Nei model (Tamura et al., 2012).

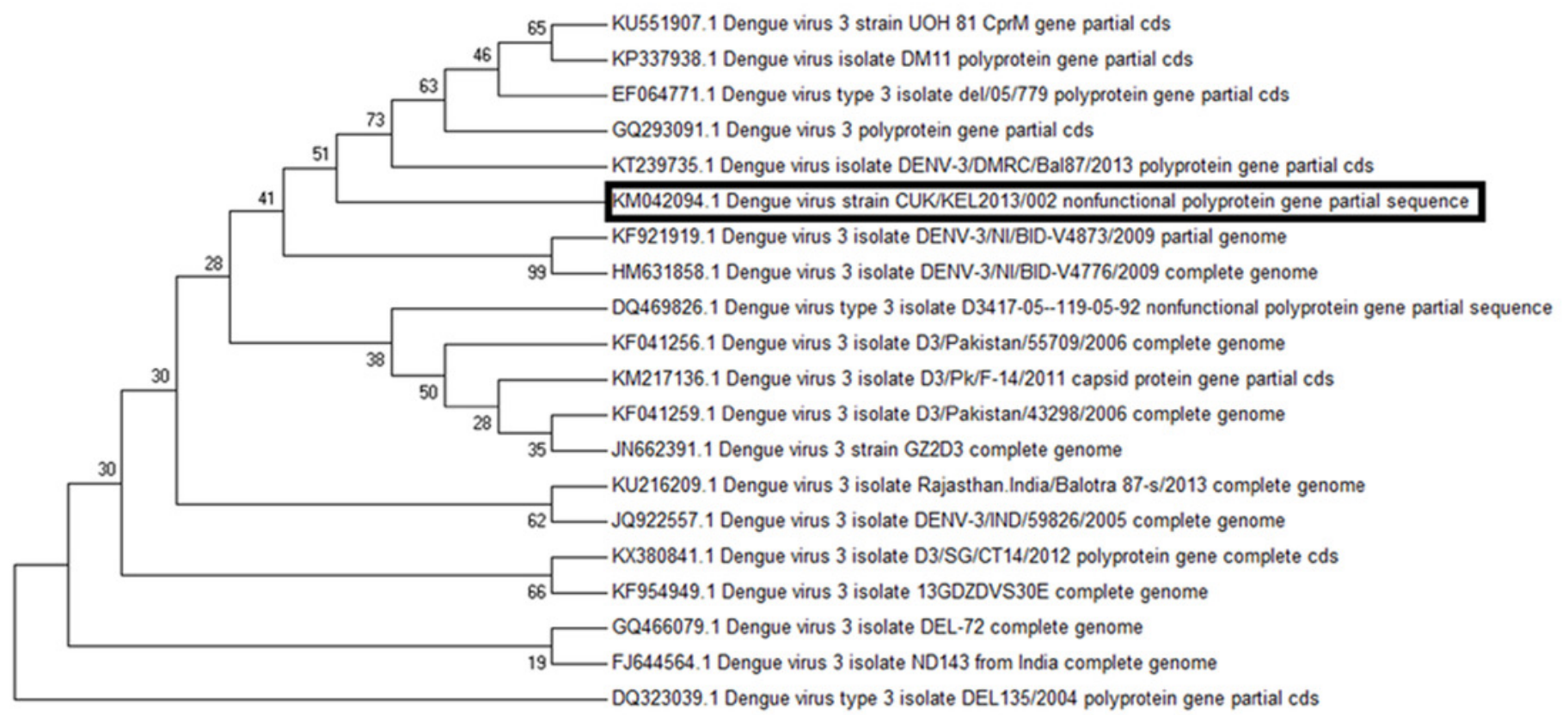




\section{Table $\mathbf{1}$ (on next page)}

Dengue infected samples analysed during the year 2013-2015.

Total 120 samples were analyzed during the years 2013, 2014 and 2015, out of which 100 samples were NS1 positive. Among the 100 samples, 26 samples were dengue viral RNA positive, out of which, three serotypes (DENV 1, 2 and 3) co-existed in eight samples, two serotypes (DENV land DENV 3) co-existed in nine samples and two serotypes (DENV2and DENV3 ) co-existed in seven samples and four co serotypes (DENV1, 2, 3 and 4) co-existed in two samples. In all cases, multiple infections were observed. However, concurrent infection of all four serotypes was only observed during 2013. 
Table 1.

\begin{tabular}{|c|c|c|c|c|}
\hline S.No & Sample description & 2013 year & 2014 year & 2015 year \\
\hline 1 & Total number of samples & 60 & 20 & 40 \\
\hline 2 & NS1 positive & 51 & 17 & 32 \\
\hline 3 & NS1 negative & 9 & 3 & 8 \\
\hline 4 & RNA positive & 13 & 3 & 10 \\
\hline 5 & Single infection & NIL & NIL & NIL \\
\hline 6 & Multiple infection & all & all & all \\
\hline 7 & DENV1 and DENV3 & 4 & 1 & 4 \\
\hline 8 & DENV2 and DENV3 & 2 & 1 & 4 \\
\hline 9 & DENV1,2 and 3 & 5 & 1 & 2 \\
\hline 10 & DENV1,2,3 and 4 & 2 & 0 & 0 \\
\hline
\end{tabular}

